\title{
3D Printing Role in Filling the Critical Gap in the Medical Supply Chain during COVID-19 Pandemic
}

\author{
Mohsen Attaran \\ School of Business and Public Administration, California State University, Bakersfield, USA \\ Email: mattaran@csub.edu
}

How to cite this paper: Attaran, $M$. (2020). 3D Printing Role in Filling the Critical Gap in the Medical Supply Chain during COVID-19 Pandemic. American Journal of Industrial and Business Management, 10, 988-1001.

https://doi.org/10.4236/ajibm.2020.105066

Received: May 7, 2020

Accepted: May 23, 2020

Published: May 26, 2020

Copyright (c) 2020 by author(s) and Scientific Research Publishing Inc. This work is licensed under the Creative Commons Attribution International License (CC BY 4.0).

http://creativecommons.org/licenses/by/4.0/

\begin{abstract}
$3 \mathrm{D}$ printing is a process of making three-dimensional objects using additive processes where layers are laid down in succession to create a complete object. Companies across the globe are actively piloting and leveraging the inherent benefits of 3D printing technology. Today, 3D printing is expanding the landscape of supply chains and providing critical medical supplies to fight the COVID-19 pandemic. This article explores 3D printing's current technologies and trends and identifies its key benefits and advantages that shape the future of many industries. Additionally, the article highlights 3D printing applications in different industries. Finally, the article investigates the crucial rolls the technology plays in filling the critical gap in the supply chains of the healthcare industry during the recent pandemic.
\end{abstract}

\section{Keywords}

3D Printing, Additive Manufacturing, Rapid Prototyping, Selective Laser Sintering (SLS), Fused Deposition Modeling (FDM), Stereolithography (SLA), Digital Light Processing (DLP), Medical Supply Chains

\section{Introduction}

The $3 \mathrm{D}$ printer works in a very similar way to the standard inkjet printer. However, instead of printing layers of ink on paper, a 3D printer uses materials to build a three-dimensional object (Berman, 2012). A variety of materials can be utilized, including metals, plastics, resins, rubbers, ceramics, glass, and concretes (Bogue, 2013). Most commercial 3D printers use a computer-aided (CAD) design to translate the design into a three-dimensional object. The design is then used by the 3D printer to deposit the layers of material (Chua et al., 2010). Many 
in the industry use the terms 3D printing and additive manufacturing interchangeably. Additive manufacturing (AM) is the broader and more all-inclusive term. Additive manufacturing refers to the technology of producing a final three-dimensional product by depositing thin layers of material upon each other (Wohlers and Gomet, 2014).

The first working 3D printer, "Stereolithography Apparatus", was created in 1984 by Chuck W. Hull of 3D Systems Corp (Bogue, 2013). In the early days, technology was costly and not feasible for the general market. In recent years, however, costs drastically decreased, allowing 3D printers to find their way in many industries, including distributed manufacturing, prototyping, and product designs (Pei, 2014), and healthcare products (Murphy \& Atala, 2014). According to a recent report by International Data Corporation (IDC), worldwide spending on 3D printing (including hardware, materials, software, and services) was $\$ 11.4$ billion in 2018. The spending is expected to exceed $\$ 13.8$ billion in 2019 and will exceed $\$ 23$ billion by 2022 , with a five-year compound annual growth rate of more than 18.0 percent. The report predicted that by 2021,40 percent of the top 2000 manufacturers would utilize 3D printing. These figures highlight a 3D market that is thriving. Drivers for the rapid growth are the reduced cost to access the technology, the emergence of higher speed 3D printers, the expanded range of materials available for 3D printing systems, and an increase in applications (Daquila and Shirer, 2018). It is estimated that in 2019, The United States will be the region with the most substantial spending on 3D printing ( $\$ 5.0$ billion), followed by Western Europe ( $\$ 3.6$ billion). These two regions will provide nearly 75 percent of all $3 \mathrm{D}$ printing spending. China will be the third-largest region with more than $\$ 1.9$ billion in spending. The rest of the world will make up less than 10 percent of global spending on 3D printing (Daquila and Shirer, 2018).

Today, 3D printing is reemerging, and it is now one of the hottest and exciting advancements in the design and marketing world. COVID-19 has transformed 3 -D printing and highlighted the importance of 3D printers and drastically increased their applications in making personal protective equipment for healthcare workers by universities, tech firms, and 3D print enthusiasts. The critical application of 3D printing, the rapid prototyping technology, allowed designs for a face mask part, ventilator component, or even a coronavirus nasal testing swab to instantly be pushed out to thousands of computers. Instead of waiting for these medical products and components to be mass-produced overseas and then shipped to hospitals, 3-D printing allowed components to be made on-demand and close by. COVID-19 pandemic has exposed vulnerabilities in global supply chains and has supported the 3-D printing industry's growth (Nikolopoulos, 2020). Individual designers with 3D printers at home are pulling open-source component designs from the internet and answering calls from local hospitals and nursing homes for medical supplies. As the coronavirus pandemic takes hold over the globe, 3D printers have helped to fill the critical gaps in the supply chain. They have ensured that the same level of face shields, nasal swabs, adap- 
tors, valves, ventilators, and other complex medical supplies continue to reach hospitals (Petch, 2020).

Section 2 explores 3D printers' current technologies and trends. Section 3 reviews $3 \mathrm{D}$ printing advantages and discusses transforming potentials of the technology. Section 4 summarizes real-world applications of 3D printing across different industries. Section 5 highlights crucial roles 3D printing plays to aid in the mass shortages of critical medical supplies as part of the response to the COVID-19 global pandemic. Section 6 provides a summary and conclusions.

\section{Technology Trends}

The technology has advanced from its roots in the production of simple plastic prototypes. Today, 3D printers produce fully functional components, including complex replacement parts, batteries, transistors, and LEDs. The capabilities of 3-D printing hardware are evolving rapidly. Next 3D printers can build more significant components and achieve greater precision and resolution faster and at lower costs. 3D printers are becoming a viable alternative to conventional manufacturing processes in an increasing number of applications (Cohen et al., 2014). 3D printers use different technologies. They all use additive processes, differing mainly in the way successive layers of matrials are built to create the final object. Figure 1 summarizes some key characteristics of the four most popular 3D printers' technologies, highlights the materials they use and industries they serve with a summary of their advantages (Attaran, 2017b). Over the coming years, we expect to see dozens of new technologies related to $3 \mathrm{D}$ printing to appear. These technologies are expanding the performance of printers along different dimensions, including speeds, range of environments, a variety of materials, versatility, and software advances (Annunziata, 2019). New 3D printers are designed to be 10 times faster than traditional printers and can operate in an uncontrolled and broader range of remote environments such as in an oil rig or an army base. 3D printers will become more versatile and will handle a growing and broader range of materials, including different metals, superalloys, nylon,

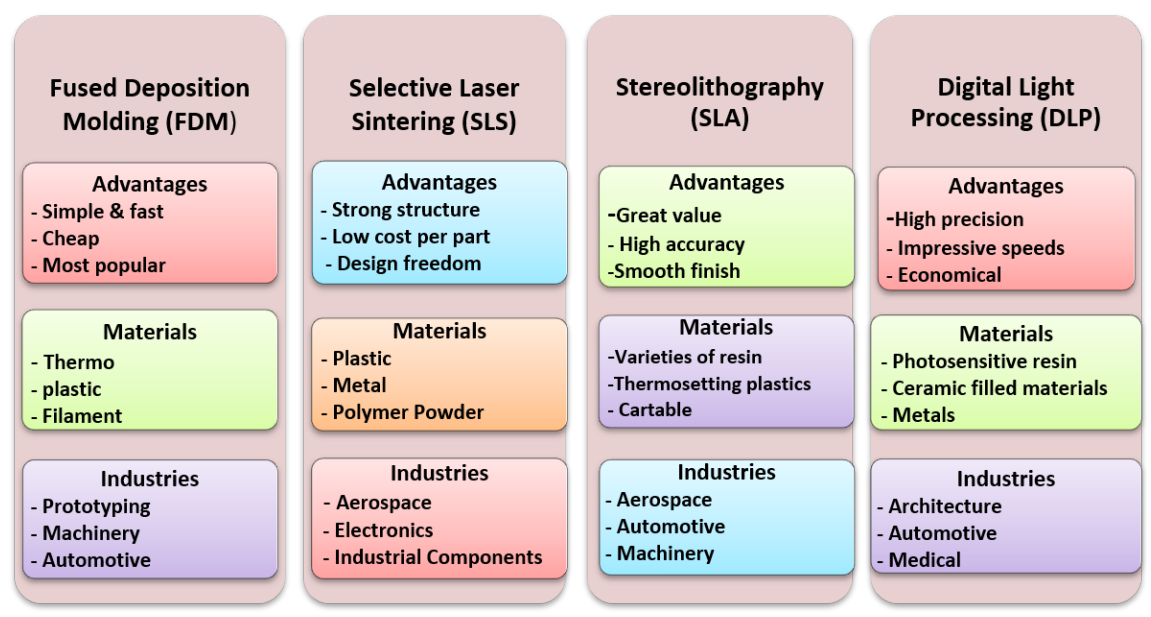

Figure 1. 3D printing technology and materials. Source: Attaran, 2017a. 
and carbon fiber. Finally, software companies are developing new software solutions that will simplify the power of 3D printing, improve security, and manufacturing precision (Annunziata, 2019).

Each 3D printing technology has its strengths and equipment and is suitable for different applications. The two popular technologies using melting or softening materials to produce the layers are Selective Laser Sintering (SLS) and Fused Deposition Modeling (FDM). SLS utilizes a high-powered laser (such as carbon dioxide laser) to fuse small particles of plastic, ceramic, metal, or glass powders into a mass that has the desired 3-dimensional shape. The technology is a popular choice among engineers for functional prototyping. This technology is accessible and in extensive use due to its ability to make low cost and reliable structured parts directly from digital CAD data. It is a simplified process for creating prototypes and even final products (Attaran, 2017a).

FDM, also called Fused Filament Fabrication (FFF), utilizes thermoplastic materials injected through indexing heated nozzles onto a platform. FDM is the most popular of all the 3D printing technologies, and it is easy to implement and can create very high-quality products. The technique is well suited for quick and low-cost prototyping of simple parts and basic proof-of-concept models (Dudek, 2013).

Another 3D printing method is called SLA or Stereolithography utilizes laser technology to cure layer-upon-layer of photopolymer resin. First, the laser hardens the resin for a layer. Then the model is lifted, and another layer is created beneath the previous. The significant advantages of SLA are the ability to create very high-quality parts with high precision and smooth finish surface detail of all plastic 3D printing technology. It is an excellent option for highly detailed prototyping, and it is widely used in a range of industries, including manufacturing, dentistry, jewelry, and education (Khorram Niaki and Nonino, 2017).

Finally, Digital Light Projection or Digital Light Processing (DLP) uses similar technology as SLA. The only significant difference is the light source used to cure the resin. DLP printers use a specially developed digital light projector screen where the projector screen flashes an image of a layer all at once. DLP printers are economical and can achieve highly specific parts (Greguric, 2018).

\section{Key Benefits and Advantages}

A key benefit of the first $3 \mathrm{D}$ printing machines was the reduction of time in product development of prototypes. Now 3D printers are poised to help many industries reduce production time, increase efficiency, and reduce costs. For example, 3D printers are being used to get prototypes into the hands of customers faster. The ability to make prototypes faster and at a lower cost enables companies to quickly test multiple configurations, reducing product-launch time to market (Cohen et al., 2014).

Following are 3D printing advantages and disruptions that shape the future of many industries (Attaran, 2017a; Khorram Niaki and Nonino, 2017): 


\subsection{Advantages}

1) Industrial Efficiency: 3D printing would make it possible for consumers to print their parts for fixing their items. Consumers can become micro-manufacturers.

2) Component Manufacturing: Industries that require low quantities of components that must be printed to precise specifications can utilize this technology.

3) Printing Complete Systems: 3D printing can print complete systems or subsystems. This multi-material capability will help since most finished products are made from more than one material.

4) Quality Improvement: 3D printing has the potential for improving quality. The technology will also help companies enhance their aftermarket services. The consumer is fast transforming into a producer-consumer as manufacturing is moving closer to the consumers.

5) Modifications and Redesigns without Penalties: 3D printing also facilitates design and innovation without time or cost penalties. It enables engineers to try multiple iterations simultaneously with minimal additional costs.

6) Increased Supply Chain Proficiency: 3D printing allows for real-time visibility to production and receipt of parts. General manufacturing time and cost are minimized through the process.

\subsection{Disruptions}

1) Mass Customization: $3 \mathrm{D}$ printing enables mass customization at a low cost. Retailers can design and personalize goods without a longer delivery time.

2) Disruptive Competitors: Many benefits of $3 \mathrm{D}$ printing could cut the cost of market entry for new players.

3) Decentralized Manufacturing: The technology could transform designs digitally, leading to a decentralization of manufacturing and reducing the time from production to sale.

4) On-Demand Manufacturing: Additive manufacturing facilitates on-demand manufacturing of replacement parts where parts could be printed in remote locations by local distributors and service providers.

5) Sustainable Environmental Impacts: $3 \mathrm{D}$ printing leaves a smaller environmental footprint. The technology generates little waste as only the needed materials are used.

\section{Real-World Applications of 3D Printing}

The first application for 3D printing was rapid prototyping that assisted with increasing time-to-market and innovation. It can be referred to as the process of quickly creating a model/prototype of a part or finished goods. This part or finished goods will be further tested and scrutinized before mass production occurs. Most commercial 3D printers have similar functionality. The printer uses a computer-aided (CAD) design to translate the design into a three-dimensional 
object. The design is then sliced into several two-dimensional plans, which instruct the 3D printer where to deposit the layers of material (Khorram Niaki and Nonino, 2017). Falling costs and improved capabilities of 3D printing are increasing the breadth and depth of application of 3D printing. The technology has improved from its roots in the production of simple plastic prototypes. Today, 3D printers produce fully functional components, including complex replacement parts, batteries, transistors, and LEDs. The capabilities of 3D printing hardware are evolving rapidly (Attaran, 2017a). Next 3D printers can build more significant components and achieve greater precision and resolution faster and at lower costs. 3D printers are becoming a viable alternative to conventional manufacturing processes in an increasing number of applications (Cohen et al., 2014).

3D printers have the potential to create complex parts and structures that were not possible before. They can allow companies to slash development time, eliminate tooling costs, and simplify production runs. Moreover, 3D printers can help companies improve the productivity of materials by removing the waste that accrues in traditional or subtractive manufacturing. These advantages could lead to profound changes in the way many things are designed, developed, produced, and supported (Cohen et al., 2014).

According to an estimate by McKinsey, various 3D applications will generate an economic impact of $\$ 230$ billion to $\$ 550$ billion per year by 2025 . International Data Corporation (IDC) reported that the leading applications for 3D printing are prototypes, parts for new products, and aftermarket parts. These three applications accounted for 45 percent of worldwide spending in 2019. Additionally, dental and medical support objects and tissue/organ/bone printing will see a five-year compound annual growth spending rate of more than 21.0 percent (Daquila and Shirer, 2018).

$3 \mathrm{D}$ printing is primarily used to make low-cost prototypes and mockups. However, the breadth of application of 3D printing continues to grow. Industries benefiting from 3D printing are briefly explained below:

1) Manufacturing: One of the first applications of $3 \mathrm{D}$ printing was in the automotive industry. The first 3D-printed car was manufactured in 2014 by Local Motors. The car was named Strati and made from an ABS carbon-fiber blend. Ford has been $3 \mathrm{D}$ printing prototypes for over 25 years to decrease design time. General Motors has been using Additive Manufacturing (AM) to make prototypes for over 20 years to speed up time-to-market and to reduce the cost involved in product development (Fish, 2011). Recently, automakers are using 3D printing technology to produce many automobile parts during the manufacturing process. According to a report by SmarTech, the consumption of 3D printing materials by the automotive industry will reach around $\$ 530$ million, and revenues will reach $\$ 2.3$ billion by 2021 (SmarTech., 2016). The technology has been pushed beyond prototyping and has advanced the way parts and tools are produced in the Aerospace industry. GE has spent over $\$ 1$ billion in 3D printing technology to meet its jet engine needs, including reducing the total number of 
engine parts from 855 to 12 , lowering the engine's weight by five percent, and improving fuel efficiency (Marren, 2017).

2) Medical: One of the first ways that $3 \mathrm{D}$ printing transformed the medical industry was fabricating custom implants, such as prosthetics and hearing aids (Berman, 2012). 3D bioprinting has been used to generate and transplant several types of tissues, including skin, bone, heart tissues, vascular grafts, other vital tissues, and organs (Murphy and Atala, 2014). Other applications of 3D printing include dental copings, body parts, reconstructing bones, and heavily damaged evidence acquired from crime scene investigations in forensic pathology (Attaran, 2017a).

3) Retail: 3D printed shoes and clothing are already in the market. 3D printed fashion and consumer goods are slowly making their way in the Retail industry (Honigman, 2014). Retailers could someday use 3D printers to allow customers to tailor products or build materials to suit their needs. In that case, retailers could own the machines, core designs, or both.

4) Others: NASA has been testing $3 \mathrm{D}$ printing in zero gravity to establish on-demand manufacturing for astronauts. This would allow parts for maintenance and repair of the international space station to be manufactured in Space (King, 2012; NASA, 2014). An additional application of 3D printing is in the novelty sector. Typically, these products have a short life span and will not remain in the market for an extended period. 3D printing can be used to reduce the risk of excess or obsolete inventory (Crandall, 2013).

5) Food Printing: 3D printing of food is becoming a reality. 3D food printers work in virtually the same way as traditional 3D printers. Most 3D food printers use extrusion printing technology and use paste-type ingredients instead of using plastic material. A syringe or cartridge holds food ingredients and printer 3D print food, layer after layer, generally through a syringe-like extruder, and create a wide range of food-from pizzas to cakes. Food printing would make it possible to create food with more complex and innovative recipes (Wegrzyn et al., 2012; Walters et al., 2011). The technology makes it possible to locate production facilities near the end customer. Therefore, the delivery of food is no longer a restriction. This results in a shortening of the customized food supply chain and savings in packaging, distribution, and overhead costs (Chen, 2016; Jia et al., 2016). 3D food printing can expand the source of available food material. Non-traditional food materials such as high fiber plant-based materials, animal-based by-products, and insects can be used by these printers to create a variety of foods (Payne et al., 2016). Food printing technology enables faster product design, customization, cost reduction, faster product testing, and more. Currently, 3D food printing is being applied in food areas such as military food, space food, elderly food, and sweets food (Godoi et al., 2016; Lipton et al., 2015).

6) Replacement Parts: $3 \mathrm{D}$ printing advantages and disruptions shape the future of many industries. 3D printing facilitates the on-demand manufacturing of replacement parts where parts could be printed in remote locations by local distributors and service providers. Furthermore, 3D printing enables mass custo- 
mization at a low cost. Retailers and manufacturers can design and personalize goods without a longer delivery time. Manufacturing reflow and supply chain transformation lead to the reduction of global freight volume. That would affect the logistics industry (Chen, 2016). When the supply chains of critical products, like medical supplies, are strained, 3D printers can play a pivotal role in producing such vital equipment when they are hard to source. For example, during the ongoing Syrian civil war and refugee crisis, sourcing of medical devices, including prostheses, has been challenging due to cost and the complications of delivering items into a warzone. $3 \mathrm{D}$ printing of prostheses significantly reduced manufacturing costs and allowed the devices to reach patients faster (Petch, 2020).

7) Altering Supply Chains and Logistics: Altering Supply Chains and Logistics-3D printing has the potential to reduce complexity in the supply chain in several ways (Kubáč and Kodym, 2017). An essential benefit of the technology lies in the consolidation of components into a single product, which leads to a reduction in inventory complexity. 3D printing could also remove the assembly and pre-assembly steps and has the potential to reduce the supplier base of the company. 3D printing is a strong enabler of product customization and can have remarkable impacts on production and distribution (Attaran, 2017b). The technology has the potential to reduce costs and increase profits by involving clients in the design and production stages and tailoring individualized offers to each customer. As a result, the supply chain can quickly react to changes in the marketplace. In summary, 3D printing can affect supply chains in many ways, including accelerated product development, reduced economic lot size, increased production flexibility, and reduced material waste (Attaran, 2017b). Adopters can rip the benefits from sourcing of materials to logistics and product distribution. Furthermore, the impact of 3D printing on the global setup of supply chains can be very disruptive. The technology has the potential to eliminate the need for both high volume production facilities and low-level assembly workers, thereby drastically reducing supply chain cost. Manufacturing can take place almost anywhere at the same cost. Thereby, it is no longer financially efficient to transport products moving across the globe to get to the customer. Manufacturing reflow and supply chain transformation lead to the reduction of global freight volume. That would affect the logistics industry (Chen, 2016).

\section{3D Printing and COVID-19}

According to a new survey of more than 1000 U.S. manufacturing companies by Thomas Industry, 60 percent of companies have already felt effects through disruptions in shipping, and many have been forced to turn down or delay new orders (Nikolopoulos, 2020). 3D printing helps to provide critical medical supplies and is an essential part of the response to the COVID-19 global epidemic. The strengths of 3D printing are that it can be anywhere, can print virtually anything, and adapt on the fly (Khorram Niaki and Nonino, 2017). Those features make it 
a capability for helping address shortages of parts related to shields, masks, and ventilators, among other things. 3D printers have played a crucial role in filling the critical gaps in the medical industry's supply chain during the coronavirus pandemic. 3D printers have been used in the design and production of personal protection equipment for healthcare workers. From protective masks to parts for ventilators, individuals, and private sectors are busy printing parts to ease the shortages of medical supplies caused by sudden demand during COVID-19 pandemic.

The 3D printing sector can play an essential role in sustaining the effort of hospital workers in the middle of the COVID-19 pandemic. As the coronavirus pandemic takes hold over the globe, some patients may require specialist respirators to take over the lungs. These respirators are in short supply, along with shields, nasal swabs, adaptors, valves, and other complex medical supplies. COVID-19 highlighted the importance of 3D printers and drastically increased their applications. Governments, universities, tech firms, professional AM providers, and 3D print enthusiasts have already begun to respond to the global crisis. They volunteered their skills to ease the pressure on supply chains and governments and aid in producing medical equipment for hospitals.

1) Governments: The European Association for Additive Manufacturing (CECIMO) has extended the call to include all AM companies within Europe to aid in producing medical equipment, including valves and ventilators for hospitals tackling the COVID-19 outbreak. The Association suggested that the Member States consider a temporary waiver of specific Medical Device Directive requirements to produce medical equipment quickly. As a result, many companies from the European 3Dprinting industry are volunteering to aid hospitals and health centers by offering their machines (Petch, 2020).

2) Universities: According to an article published in The Optical Society journal, researchers from several universities have produced a 3D printed highresolution digital holographic microscope (Essop, 2019). The microscope is made entirely from 3D printed parts and commonly found optical components. It could potentially be utilized to diagnose diseases like sickle cell disease, malaria, diabetes, and others. The simplicity and low cost of constructing the instrument could increase access to low-cost medical diagnostic testing where there is limited access to health care and few high-tech diagnostic facilities. Several colleges from Idaho State University (ISU), including the College of Science and Engineering and the College of Technology, have teamed up and used 3D printers to answer the call to meet the needs for personal protection equipment (Idaho State University, 2020). The group produced components for face shields, face masks and other personal protective equipment for use by ISU clinics, ISU health care workers, and emergency responders and health care workers. Elsewhere, researchers at Australia's RMIT University designed an open-source 3D printed "clip-on" filter that can turn smartphone cameras into a powerful microscope (Essop, 2019). Another research group at the UK's University of Bath 
developed a 3D printable microscope prototype. The microscope is a high-quality instrument for serious microscopy and is intended to increase the accessibility of educational and functional scientific tools (Essop, 2019). Under the guidance of medical professionals, graduate students, and staff at Purdue University have redesigned and manufactured complex fittings for ventilators. They are actively producing 3D-printed parts for face shields and safety glasses (Reinke, 2020).

3) 3D Printing Firms: 3D printing firms are also responding to the COVID-19 outbreak by volunteering their skills and putting their expertise to good use. Companies like Stratasys, Carbon, and Shapeways are rushing to rapidly-produce much-needed ventilator components, face masks, and medical test equipment during the pandemic (Petch, 2020). Materialize, a global provider of 3D printing services, has released files for a 3D printed add-on, hands-free door handle attachment that is free to download. The add-on allows users to open most modern doors without touching door handles and using their elbows. Door handles are subjected to a lot of physical contacts, especially in public places such as offices and hospitals, and are a hotspot for microbes. This 3D printed hands-free door handle attachment, can be made in under 4 hours, and alleviate Coronavirus transmission via one of the most common mediums (Petch, 2020). California-based Airwolf3D have volunteered their fleet of 3D printers to manufacture respirator valves and custom medical components (Petch, 2020). A Chinese manufacturer has offered its in-house print farm of 200 3D printers to mass produce safety goggles for healthcare professionals in China. The company has fabricated more than 5000 pairs of 3D printed safety goggles and donated to Chinese hospitals. The company aims to increase daily production to 10,000 pairs in the coming a short time (Petch, 2020). Winsun, a Chinese architectural $3 \mathrm{D}$ printing company, has dispatched $153 \mathrm{D}$ printed quarantine booths to a Hospital just outside Wuhan China. In the first few weeks of Coronavirus spread hospital beds quickly became a pressing issue. Winsun Used solid urban construction waste and 3D-printed and fabricated small individual quarantine booths. The rooms' interiors are decorated and have their water and electricity supplies (Petch, 2020). Stratasys, a 3D printer manufacturer, has mobilized its global 3D printing resources to 3D print 5000 full-face shields in under a week to respond to the COVID-19 pandemic. The face shield will be provided a no cost to medical personnel (Petch, 2020).

4) Engineering Firms: An Italian hospital with 250 Coronavirus patients run out of the respiratory valves required to connect the patients to the machines. The hospital quickly found itself in a crisis as the original supplier was unable to meet the sudden high demand. An Italian engineering firm used in-house SLS 3D printers to produce 100 respirator valves in 24 hours, met the hospital's demands, and, resultantly, patients' lives were saved (Essop, 2020).

5) Automakers: Many automakers have put vehicle production on hold over concerns about spreading Coronavirus. Many of them are volunteering their fleet of 3D printers for the fabrication of medical components. 3D printing lets automakers get a proof of concept and initial design fairly quickly and make in- 
cremental improvements based on recipient feedback before mass production. GM, Ford, and Tesla joined the fight against pandemic and applied their manufacturing muscle to fabricate ventilators and personal protective equipment in considerable numbers to supply more of this vital equipment to the medical community (O'Kane, 2020). Volkswagen has announced it will adapt its more than 125 industrial 3D printers to produce in-demand medical equipment to tackle the COVID-19 pandemic (Petch, 2020). Finally, Italian sports car manufacturer Ferrari begins 3D printing production of respirator values and fittings for protective masks to ease the shortages of these needed supplies for the healthcare professionals (Petch, 2020).

\section{Summary and Conclusion}

This study reviewed 3D printing technologies, discussed several advantages of this emerging technology, summarized the significant effects of 3D printing on different industries, and underlined significant factors driving high demand for the technology during the recent pandemic. The $3 \mathrm{D}$ printing process is more straightforward and eliminates many steps used in traditional manufacturing. The technology enables faster product design, customization, cost reduction, faster product testing, and more. Industries, including medical, dental, aerospace, and retailers, are using 3D printing technology successfully in the areas of product and tool development.

Several factors are enhancing the popularity of the 3D printing industry. The most significant factor is the shortages of medical supplies during the recent pandemic. In the past several months, many companies were affected through disruptions in shipping, and many have been forced to turn down or delay new orders. COVID-19 highlighted the need to diversify supply chains and reduce the vulnerability of different industries, specially the medical industry. This paper identified and examined the significant impact 3D printers have had in printing parts to ease the shortages of medical supplies caused by sudden demand during the COVID-19 pandemic.

\section{The Future}

3D printers are still an emerging technology that must overcome several challenges, including cost, and speed. However, 3D printers are getting better every year. The future of 3D printing is bright. The technology will have a massive impact on the global supply chains, giving customers a significant degree of freedom in choosing when and where to manufacture parts.

\section{Practical Implications}

Based on the business applications presented in this paper, practitioners will learn 3D printing use cases and business potentials for different industries, strategic values and challenges addressed by $3 \mathrm{D}$ printing, and critical roles the technology plays in the healthcare industry's supply chain during COVID-19 global 
pandemic.

\section{Research Limitations}

Several limitations of this research should be considered. For example, some impacts of 3D printers on the medical supply chain are not exclusive. Other manufacturing technologies can achieve these impacts. Additionally, this paper summarized these impacts using a literature review. Future extension of this research will need to emphasize the potential impacts with quantitative measures and add some non-3D printing companies.

\section{Conflicts of Interest}

The author declares no conflicts of interest regarding the publication of this paper.

\section{References}

Annunziata, M. (2019). 2020: The Year Ahead in 3D Printing. Forbs. https://www.forbes.com/sites/marcoannunziata/2019/12/17/2020-the-year-ahead-in-3d -printing/\#2e761b16407f

Attaran, M. (2017a). The Rise of 3-D Printing: The Advantages of Additive Manufacturing over Traditional Manufacturing. Business Horizons, 60, 677-688. https://doi.org/10.1016/j.bushor.2017.05.011

Attaran, M. (2017b). Additive Manufacturing: The Most Promising Technology to Alter Supply Chain and Logistics. Journal of Service Science and Management, Special Issue in Supply Chain Management, 10, 189-205. https://doi.org/10.4236/jssm.2017.103017

Berman, B. (2012). 3D Printing: The New Industrial Revolution. Business Horizons, 55, 155-162. https://doi.org/10.1016/j.bushor.2011.11.003

Bogue, R. (2013). 3D Printing: The Dawn of a New Era in Manufacturing? Assembly Automation, 33, 307-311. https://doi.org/10.1108/AA-06-2013-055

Chen, Z. (2016). Research on the Impact of 3D Printing on the International Supply Chain. Advances in Materials Science and Engineering, 2016, Article ID: 4173873. https://doi.org/10.1155/2016/4173873

Chua, C. K., Leong, K. F., \& Lim, C. S. (2010). Rapid Prototyping: Principles and Applications (3rd ed.). Singapore: World Scientific Publishing Company. https://doi.org/10.1142/6665

Cohen, D., Sargeant, M., \& Somers, K. (2014). 3-D Printing Takes Shape. McKinsey Quarterly, January.

https://www.mckinsey.com/business-functions/operations/our-insights/3-d-printing-ta kes-shape

Crandall, R. (2013). Where Will Additive Manufacturing Take Us? APICS, Jan.-Feb. https://www.apics.org/apics-for-individuals/apics-magazine-home/magazine-detail-pa ge/2013/01/28/where-will-additive-manufacturing-take-us

Daquila, M., \& Shirer, M. (2018). IDC Forecasts Worldwide Spending on 3D Printing to Reach \$23 Billion in 2022.

https://www.businesswire.com/news/home/20180803005338/en/IDC-Forecasts-World wide-Spending-3D-Printing-Reach

Dudek, P. (2013). FDM 3D Printing Technology in Manufacturing Composite Elements. 
Archive of Metallurgy and Materials, 58, 1415-1418.

https://doi.org/10.2478/amm-2013-0186

Essop, A. (2019). High Resolution 3D Printed Microscope Could Help Detect Diseases in Developing Countries. 3D Printing Industry.

https://3dprintingindustry.com/news/high-resolution-3d-printed-microscope-could-he lp-detect-diseases-in-developing-countries-154556

Essop, A. (2020). Hospital in Italy Turns to 3D Printing to Save Live of Coronavirus Patients. 3D Printing Industry.

https://3dprintingindustry.com/news/hospital-in-italy-turns-to-3d-printing-to-save-liv es-of-coronavirus-patients-169136

Fish, E. (2011). Rapid Prototyping How It's Done at GM: Additive Manufacturing Technology Is Helping the Automaker Reduce Product Development Times and Costs. Automotive Design and Production, 123, 46-48.

Godoi, F. C., Prakash, S., \& Bhandari, B. R. (2016). 3D Printing Technologies Applied for Food Design: Status and Prospects. Journal of Food Engineering, 179, 44-54. https://doi.org/10.1016/j.jfoodeng.2016.01.025

Greguric, L. (2018). What Is a DLP Printer? Simply Explained. All3DP. https://all3dp.com/2/what-is-a-dlp-3d-printer-3d-printing-simply-explained

Honigman, B. (2014). How 3D Printing Is Reinventing Retail. Forbes, 3 June.

Idaho State University (2020). Idaho State University Using 3D Printers to Produce Coronavirus Personal Protective Equipment.

https://www.idahostatejournal.com/community/idaho-state-university-using-3d-printe rs-to-produce-coronavirus-personal-protective-equipment/article_7abbced8-2626-526

3-ab57-3bfb7a2c48ec.html

Jia, F., Wang, X., Mustafee, N., \& Hao, L. (2016). Investigating the Feasibility of Supply Chain-Centric Business Models in 3D Chocolate Printing: A Simulation Study. Technological Forecasting and Social Change, 102, 202-213.

https://doi.org/10.1016/j.techfore.2015.07.026

Khorram Niaki, M., \& Nonino, F. (2017). Impact of Additive Manufacturing on Business Competitiveness: A Multiple Case Study. Journal of Manufacturing Technology Management, 28, 56-74. https://doi.org/10.1108/JMTM-01-2016-0001

King, R. (2012). 3D Printing Coming to the Manufacturing Space and Outer Space. York New: Bloomberg LP.

Kubáč, L., \& Kodym, O. (2017). The Impact of 3D Printing Technology on Supply Chain. MATEC Web of Conferences, 134, Article ID: 00027.

https://doi.org/10.1051/matecconf/201713400027

https://www.researchgate.net/publication/320927657_The_Impact_of_3D_Printing_Te chnology_on_Supply_Chain

Lipton, J., Cutler, M., Nigl, F., Cohen, D., \& Lipson, H. (2015). Additive Manufacturing for the Food Industry. Trends in Food Science \& Technology, 43, 114-123. https://doi.org/10.1016/j.tifs.2015.02.004

Marren, T. (2017). 3D Printing Could Take the Aviation to New Heights. Thomas Industry.

https://www.thomasnet.com/insights/3d-printing-could-take-aviation-to-new-heights

Murphy, S. V., \& Atala, A. (2014). 3-D Bioprinting of Tissues and Organs. Nature Biotechnology, 32, 773-785. https://doi.org/10.1038/nbt.2958

NASA (2014). Space Tools on Demand: 3D Printing in Zero G. Washington DC: National Aeronautics and Space Administration. 
http://www.nasa.gov/sites/default/files/files/3D_Printing-v3.pdf

Nikolopoulos, S. (2020). Expanding the Landscape of the Supply Chain in Light of COVID19, Tariffs. Thomas Industry.

https://www.thomasnet.com/insights/expanding-the-landscape-of-the-supply-chain-in -light-of-covid-19-tariffs

O'Kane, S. (2020). Ford Joins Tesla and GM in Helping with Ventilator and Mask Supply. The Verge.

https://www.theverge.com/2020/3/24/21192282/ford-tesla-gm-elon-musk-coronavirusmasks-ventilators-pandemic

Payne, C. L., Dobermann, D., Forkes, A., House, J., Josephs, J., McBride, A., Müller, A., Quilliam, R. S., \& Soares, S. (2016). Insects as Food and Feed: European Perspectives on Recent Research and Future Priorities. Journal of Insects as Food and Feed, 2, 269-276. https://doi.org/10.3920/JIFF2016.0011

Pei, E. (2014). 4D Printing: Dawn of an Emerging Technology Cycle. Assembly Automation, 34, 310-314. https://doi.org/10.1108/AA-07-2014-062

Petch, M. (2020). 3D Printing Community Responds to COVID-19 and Coronavirus Resources. 3D Printing Industry.

https://3dprintingindustry.com/news/3d-printing-community-responds-to-covid-19-a nd-coronavirus-resources-169143

Reinke, J. (2020). Team of Purdue University Volunteers Aid in COVID-19 Medical Supply Production. Thomas Industry Update.

https://www.thomasnet.com/insights/team-of-purdue-volunteers-aid-in-medical-suppl y-production

SmarTech. (2016). Revenues from Additive Manufacturing in the Automotive Industry Will Reach \$2.3 Billion by 2021. Charlottesville, VA: SmarTech Markets Publishing. https://www.globenewswire.com/news-release/2016/09/22/1211309/0/en/SmarTech-Pu blishing-Report-Revenues-From-Additive-Manufacturing-in-the-Automotive-Industry-Wil 1-Reach-2-3-Billion-by-2021.html

Walters, P., Huson, D., \& Southerland, D. (2011). Edible 3D Printing. In Proceedings of 27th International Conference on Digital Printing Technologies (pp. 819-822). Minnesota.

Wegrzyn, T. F., Golding, M., \& Archer, R. H. (2012). Food Layered Manufacture: A New Process for Constructing Solid Foods. Trends in Food Science \& Technology, 27, 66-72. https://doi.org/10.1016/j.tifs.2012.04.006

Wohlers, T., \& Gorne, T. (2014). History of Additive Manufacturing. Wohlers Report, Fort Collins, CO: Wohlers Associates, Inc.

http://www.wohlersassociates.com/history2014.pdf 